\title{
Ethical Stewardship: Designing Serious Games Seriously
}

\author{
Alyea Sandovar ${ }^{1}$, Eelco Braad ${ }^{2}$, Alexander Streicher $^{3}$, Heinrich Söbke $^{4}$ \\ ${ }^{1}$ School of Leadership Development, Fielding Graduate University, Santa Barbara, California, \\ United States \\ asandovar@email.fielding.edu \\ ${ }^{2}$ School of Communication, Media and IT, Hanze University of Applied Sciences, Groningen, \\ The Netherlands \\ e.p.braadepl.hanze.nl \\ ${ }^{3}$ Fraunhofer IOSB, Karlsruhe, Germany \\ alexander.streicherdiosb.fraunhofer.de \\ ${ }^{4}$ Bauhaus-Institute for Infrastructure Solutions, Bauhaus-Universität Weimar, Germany \\ heinrich.soebke@uni-weimar.de
}

\begin{abstract}
In this chapter, we propose an ethical framework for serious game design, which we term the Ecosystem for Designing Games Ethically (EDGE). EDGE expands on Zagal's categorization of ethical areas in game design by incorporating the different contexts of design and their use. In addition, we leverage these contexts to suggest four guidelines that support Ethical Stewardship in serious game design. We conclude by discussing a number of specific areas in which ethics plays a role in serious game design. These include games in (a) a military context, (b) the consideration of privacy issues, and (c) the evaluation of game design choices.
\end{abstract}

Keywords: serious game design, information ethics, ethical stewardship, military games, educational games, ethical intelligence, design politics, values in design, data privacy, video game design, values at play, game design values.

\section{Introduction}

Games themselves are complex cultural artifacts and designed systems - they are designed objects [1]. So, too, is the study of digital games ethics-it is as complex and nuanced as are games. Often ethics and digital games are associated with the violence in games and subsequent aggressive behavior in players $[2,3,4]$. This association solely focuses on how the players are implicated by the artifact, that is, by the game- not on the ethics of the development process, the industry, nor on the developers.

As an information system, the game experience is in part defined by the design and in part by the player's interaction with the design of the game [1]. A comprehensive 
description is illustrated by Zagal [5] who makes a strong case for the consideration of certain variables (Table 1$)$.

Table 1. Zagal's ethical variables

\begin{tabular}{|l|l|l|l|}
\hline $\begin{array}{l}\text { The cultural arti- } \\
\text { fact }\end{array}$ & Business Ethics & Ethical Play & Frameworks \\
\hline $\begin{array}{l}\text { The cultural } \\
\text { artifact itself - is } \\
\text { the game good or } \\
\text { bad? }\end{array}$ & $\begin{array}{l}\text { What does it mean } \\
\text { to create the game } \\
\text { ethically? }\end{array}$ & $\begin{array}{l}\text { What does it mean } \\
\text { to play } \\
\text { fair/ethically? }\end{array}$ & $\begin{array}{l}\text { What actions do } \\
\text { games define for } \\
\text { the player? }\end{array}$ \\
\hline
\end{tabular}

Each of these variables reflect aspects of digital games, including serious games. For example, in a learning history game by the Danish Studio Serious Games Interactive, Playing History 2 - Slave Trade (H2ST), players are slaves tasked to enslave others by their owner. Each of Zagal's variables can be considered in the following manner in H2ST: As a cultural artefact — an object created by humans which informs about the object's culture and use-is H2ST a good or bad game? After all, it is a game designed to question moral choices in the player, such as collecting slaves and loading them into a ship. The player is a slave herself and is being forced to place others in a similar position. In addition, the game uses appealing visuals such as a cartoon-like mouse and a friendly dragon to draw the player into performing these acts. Is a game which elicits such conduct good or bad? Next, the business ethics can also be evaluated. What were the business motivations to create such a game? What are the moral implications of selling a game that portrays a sensitive aspect of human history? Is this perception influenced by the location of the company? The game company, Serious Game Interactive, garnered media attention with a questionable play mechanic in which the slaves needed to be stored in the ship in a Tetris-like manner (see Fig. 1). While it is historically accurate that slaves were treated as cargo, is it ethical for a company to address the topic in such a manner? 


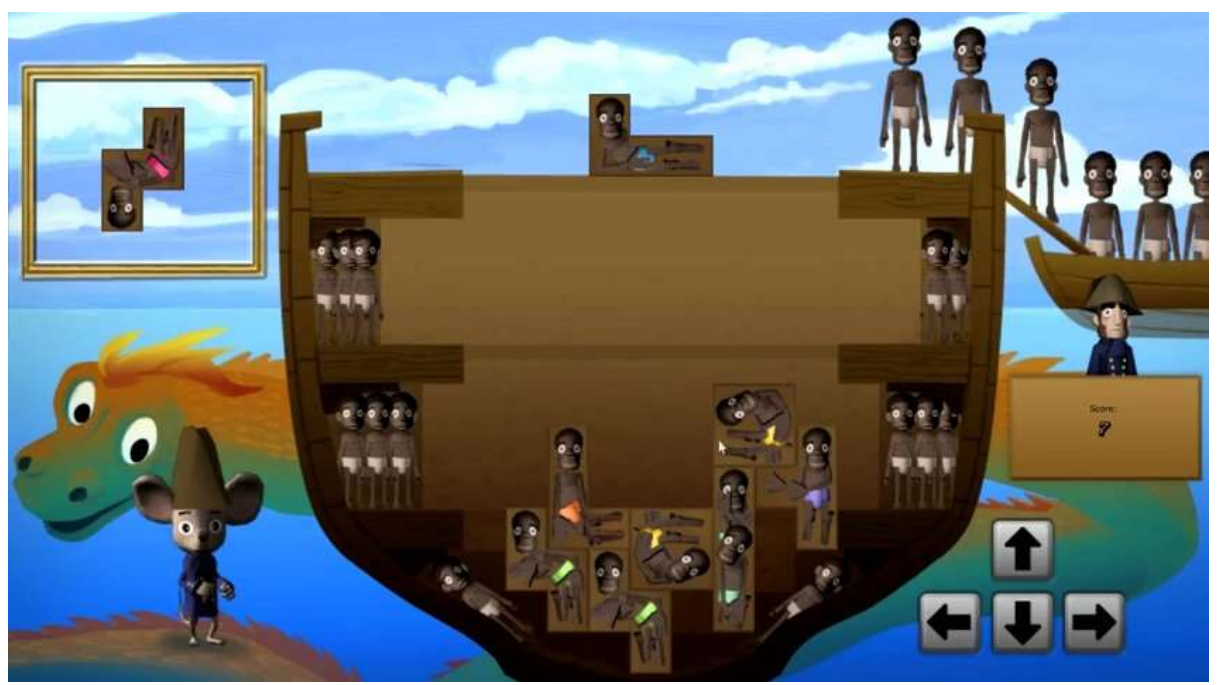

Fig. 1. Playing History 2 - Tetris Gameplay (2013).

Ethical play can showcase players' relationships to one another and the moral boundaries they are willing to cross or not. A player might choose to play the role of the slave with an intention to do as little as demanded of them to escape later and free others, for example. Frameworks are defined by the design of the game itself. The player might decide to play ethically (be an ethical player), but if the game parameters are such that unethical behavior is rewarded and ethical behavior is not, then the player is limited in her ethical play.

Entertainment games are designed for engagement and achievement, sometimes with the goal of increasing profit margins. By contrast, serious games aim to provoke thinking, elicit self-awareness, simulate experiences, support healing, or engage players in learning. The vision of serious game designers is intentional and goal oriented. Often with the to elicit behaviour change in the player. Such a focus requires a higher degree of moral intelligence in design. Thus this chapter proposes a process that enhances ethical intelligence in serious games. We term the framework for the ethical design of serious games Ecosystem for Designing Games Ethically (EDGE) and its corresponding guidelines, Ethical Stewardship.

\section{$2 \quad$ An Ethical Framework}

This chapter expands upon Zagal's variables [5] to include the designer and the context of use into the development process (Figure 2). The integration of the designer [10,11] and the context of use into Zagal's [5] variables, creates a more complete picture of the ethical ecosystem navigated in games. We use the MDA model (a formal approach to game design that includes mechanics, dynamics, and aesthetics) as a way to organize these variables into what we term an Ecosystem for Designing Games Ethically (EDGE) (see Fig. 2). This ecosystem serves as an organizing framework for this chapter, which 
is divided into five sections as follows: the two contexts of design and use, and the negotiations that occur between the designer, the serious game and the player.

Why then include the game designer herself as a variable in the framework? Discussing the ethics of digital games can include the concept that morality in games may be informed by a game designer's ethical perspective. In addition, except for business ethics, Zagal's [5] variables address aspects of post-production (after the game is created). Information systems literature offers serious games a bridge that supports the inclusion of the designer as an ethical variable. The information system field places the emphasis of ethics on pre-production (design) and extends a consideration of morality to values and beliefs in design [6,7]. If digital games reflect ethical perspectives of those who design them, then digital game ethics would do well to expand on current definitions, by including game designer's ethical perspectives that inform: (a) ethical play, (b) actions of the player, and (c) the game itself. For example, it might be challenging to fully address how race informs the degree of ethical play for the player in H2ST, if the ethical evaluation of the game does not also include an exploration of the designers and their values. For this reason, scholars in digital games ethics now address how a game designer's values inform design $[8,9]$.

In addition to the designer and the player, two important contexts should also be considered. Within the context of design, the designer makes the design choices that define the game. Within the context of use, the player interacts with the game. Much literature is already devoted to the ethics in the games themselves, in game play, and to the ethical considerations by players $[1,5,8]$. However, very little attention is given to the design contexts and the designers themselves. Within the scholarly literature of game design, there is a paucity of attention focused on the game creation process [12] and on serious games. The need for focus on these aspects is recent and has to do with the accessibility to game developer studios (including serious game studios); many have secrecy clauses and intellectual property (IP) that prevent any collected data to be used in a study, because its publication could be detrimental to the studio [13]. This section will focus on gamework, areas that refer to the development of the game including: context of design, context of use and the designers. 


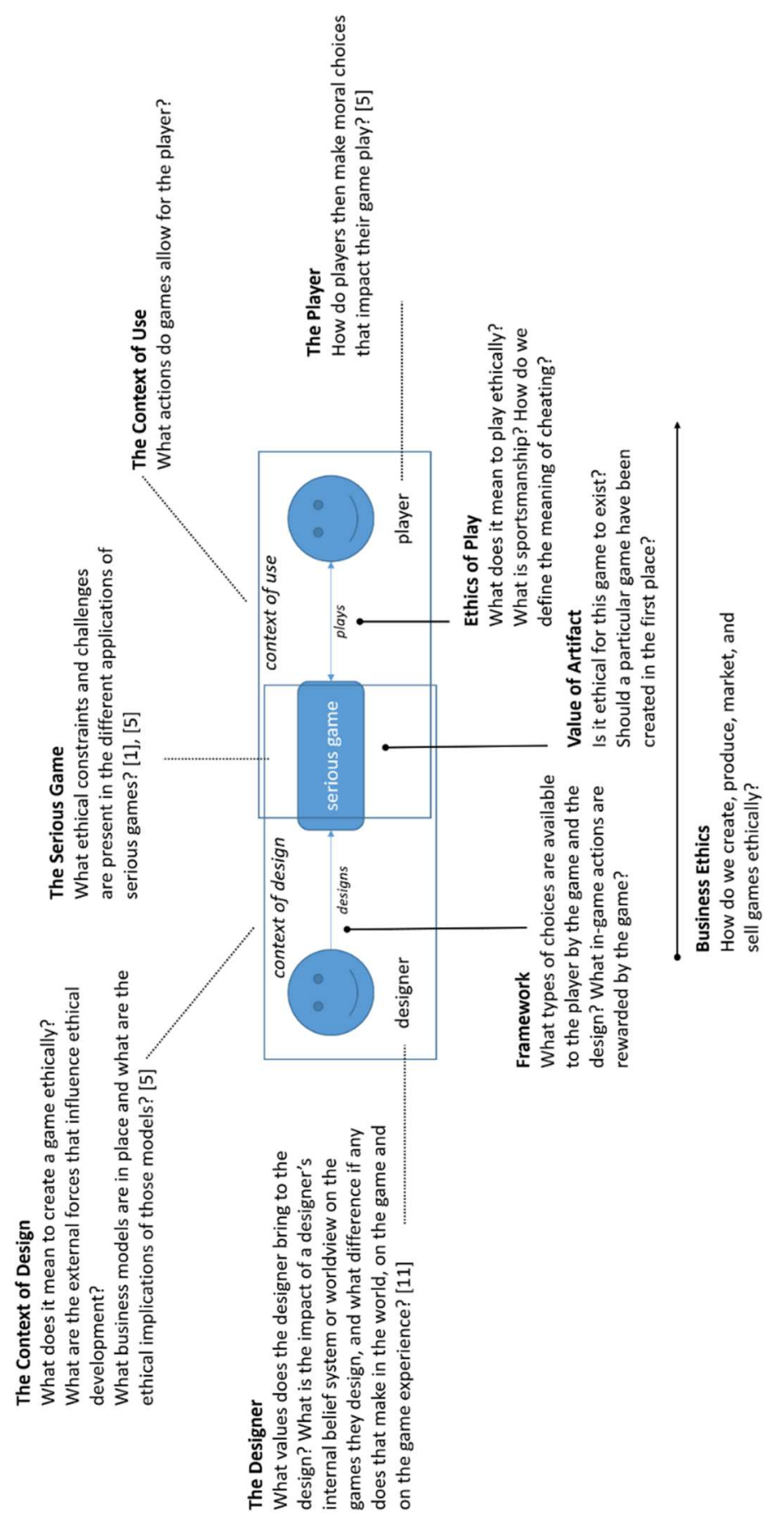

Fig. 2. Ecosystem for Designing Games Ethically (EDGE). 


\section{The Designer}

As designed software systems, games are shaped by Information and Communication Technologies (ICTs), including their historical focus on human values. The domain of computer information ethics has incorporated human values into product design since its inception [6]. To this end, there has been an emphasis to develop frameworks that ensure that moral and ethical values become integrated in the design and development of information and communication technologies (ICTs), such as digital games. These frameworks include Value Sensitive Design (VSD) [14], Worth Centered Design (WCD) [15], and Values at Play (VAP) [8,16]. Each of these frameworks attempts to influence the design of technologies by bringing moral and ethical intelligence to the creation of technologies and other cultural artifacts [17]. Scholars of information ethics (IE) also claim that technologies designed by people from a particular culture reflect the values and communication principles of the people who created them $[8,18,19]$.

This is particularly important for serious games as game designers are tasked with an agenda and a goal. If biases are embedded in the design, then unknowingly, designers could promote what Battiste [20] names a subtle cognitive imperialism. Through digital game development, designers validate their own knowledge base, and cognitively disclaim other knowledge bases and values - thus maintaining the primacy of the one language, one culture and one cultural frame [20]. This knowledge base may also be validated by those who hold the vision of the game (including designers, producers and creative directors). The example of H2ST illustrates this type of imperialism. While the scenario appeared historically accurate to the Western developers, some of the representations in the game were insensitive.

Designers therefore have a choice in the selection and presentation of the game content, and the interactions and choices that the game offers or does not offer to its users. These design choices need to be in line with the overarching purpose of the game; designers ought to consider what is conveyed with the gameplay, narrative, and the visual elements of the game. For example, in America's Army, a multiplayer first-person shooter, it is not possible to play as the enemy-a specific and deliberate design choice that allows particular content, while limiting access to other views. How then can serious game designers balance project and design demands, with ethical stewardship? The next section facilitates an understanding of ethics in design.

\subsection{Ethical Considerations in Design}

It has become clear that ethical considerations are an important aspect of designing serious games. While similar design choices need to be addressed in the production of other media, such as film and writing, the interactive aspect of games complicates the considerations that must be made. In addition to which content to present, and in what way to present it, a designer must also attend to the choices a player can make within the game, and how these choices are rewarded within the game. For example, design dilemmas exist in which roles a player is allowed to play, and which actions are available to the player within a role. An example can be found in Assassin's Creed, a series 
of action-adventure games in a fictional historical setting. The series does not contain playable female characters - even when history provides ample examples of strong female roles [21,22]. These examples demonstrate that in the presentation of the environment to play in, and what options are available to a player, ethics are at play.

As all designers do, game designers can anticipate the effects brought forward by the artefacts they create and can become accountable for their designs. However, there are a number of barriers that stand in the way of adopting this stance as regular practice. First, there is tension between ethical considerations for the design and the project constraints such as time and budget. Anticipating possible effects beyond the intended goals of the serious game is often low priority in an already complex process. Second, within game studios it is not common practice to include the ethical perspective in design. In addition, the game development literature does not include ethical thinking into the game design process nor does the literature point to the available frameworks that support ethical thinking in design $[1,5,8]$. For a nascent serious game developer, there is little material to guide their decision-making process from an ethical perspective. For example, of the current leading game design books none addresses ethics in any chapter. We propose that ethics could easily be discussed in parallel with introductions to the different stages of the game development cycle (concepting, prototyping, building and evaluating). Third, and last, the game industry at large, whether focused on serious games or entertainment games, is a relatively young field and considering the intended and unintended effects of the games produced is only starting to become regular practice. That said, there are some ways in which the designer can attempt to bring moral and ethical intelligence through design. We refer to these self-reflective and practice guidelines as Ethical Stewardship (ES) in design. These guidelines are modeled after Deardorff's intercultural competence model [23] and design models from information ethics [10].

\subsection{Ethical Stewardship in Design}

Ethical Stewardship in design requires a shift in the game designer's internal state to a reflective stance. This is achieved through: (a) developing requisite attitudes (such as respect, openness, and curiosity) which serve as the basic unit of Ethical Stewardship, (b) critical reflection on values, (c) developing ethical knowledge and skills through bricolage, and (d) developing an informed frame of reference through understanding stakeholders. The last level produces a desired external outcome which leads to more reflection in design.

1. Ethical stewardship requires developing requisite attitudes as design practice. In intercultural communication, requisite attitudes of respect, openness and curiosity comprise basic units of a reflective stance [23]. Each of these attitudes are defined in the following manner: (a) Respect refers to valuing other perspectives; (b) openness refers to the ability to withhold judgment; and (c) curiosity refers the ability to tolerate ambiguity. We propose to adopt these same attitudes as a starting point for ES. One way to increase designers' openness and curiosity is to actively seek out and include existing ethical tools [24]. One such tool is Taylor and Dempsey's RPG game, Ethical Quest 
[25]. Through the roleplaying mechanics in Ethical Quest members of a design team learn to define their own ethical stance. The goal of Ethical Quest is to collaboratively define a code of ethics with other players. The challenge however, is that players must negotiate a code of ethics while role playing a character that is part of a design team (designer, programmer, manager, player, etc.). In ethical stewardship, a designer can include these types of tools to support their development of requisite attitudes.

2. Ethical stewardship requires critical reflection on values. A model that supports game designer reflection on their personal ethical frameworks and values during design is Values at Play (VAP) designed by Mary Flanagan and colleagues [10]. Values at Play is an iterative process that designers utilize to discover, translate and verify their values within their game design. One tool developed by the VAP team is the Grow A Game cards which provide a method for designers to critical analyze and think about how to incorporate certain values into a game. Through this exercise designers gain a deeper understanding of how including values in a game from the beginning alters the game design. Another method for designers to develop critical reflection about values is to build towards understanding the influence of their own historical context on their design. To gain critical reflection, a game designer would employ a variety of interaction design tools usually used with users for self-understanding, including cultural analysis, collages and cognitive mapping [26]. By doing so she would understand better her own history and background informs her values and her worldview.

3. Ethical stewardship requires bricolage. Ethical knowledge and understanding requires intellectual bricolage [27] — what Claude Levi-Strauss calls "making do with what is at hand" [28]. A bricoleur may gather materials, knowledge and tools for future use without knowing when these will be used. In cultural anthropology the concept of bricolage occurs when new ideologies emerge from current myths and social realities. Bricolage thinking involves being resourceful and adaptable within a given context. Ethical bricoleurs: (a) are flexible and responsive - they employ a variety of research methods to gain a deeper understanding of users; (b) remain intellectually informed and stay abreast of interpretive paradigms; and (c) are multi-skilled and technically competent by enlisting different tools for gathering user information (interviews, focus groups, etc.) Additionally, a bricoleur should understand and be informed about the variety of methods available to gather diverse user data.

To gain deeper ethical knowledge in design, a game designer employing bricolage would gather materials and resources that would support a deeper understanding of differing perspectives in design. There is a Dutch saying, "vreemde ogen dwingen," which roughly translates to "strange eyes have stronger voices than eyes that look through familiar glasses." Such a saying illustrates the importance of keeping an open mind to include perspectives outside of our own. For example, ethical designers can include non-Western tools as a means for including broader perspectives. While the dichotomy of Western and non-Western is a Western construct itself [29], non-Western refers to systems of thoughts outside of European traditions including, for example, African, African American, Asian, Latin American, and indigenous populations [30]. Other design fields, like architecture, have been successful at introducing diversity in design by 
including examples of non-Western designers in their work and learning from the contributions of "invisible designers" [30,31]. Western game design process could benefit not only from the inclusion of collaborative methods during design. For example, if game designers were to develop a game based on a historical event, all those involved in the project would learn about the geography, people and history of the event from multiple perspectives [32]. When creating a game based on the U.S. Gold Rush, for example, designers would incorporate learning about the history from the perspective of indigenous nations, and not solely as accounted historically in Western texts [33].

4. Ethical stewardship requires including stakeholders as co-creators of games from the beginning. Developing an informed frame of reference requires an understanding of the people for whom the game is intended for. While it is common practice to include players in the development cycle to test a game, we propose including all appropriate stakeholders from the beginning. This approach fits well with the user-centered design process. As the design process should inherently assess and address the user, several methods supporting user advocacy have been developed and widely adopted. These approaches range from evaluating game prototypes with focus groups representing the target audience (passive participation), to actively including end users in the design team (participatory design or co-creation). Game designers can partner with stakeholders to co-create the designs, rather than perceive stakeholders in subject position, participating solely as "inputters," not partners. Furthermore, the type of stakeholder that contributes to the design of game can be selected for their skill and their creative capacity [34]. For example, in the development of an educational game to be used in schools, it is important to include teachers and parents as part of the design team.

Additionally, inclusion of co-creating with stakeholders requires much more than an implementation of design strategies, and instead asks the designer to become a facilitator of the relational process [35]. Intellectual facilitation is based upon the notion that well-understood design practices might not be appropriate for a given audience, and instead requires a mutual learning process between designer and user [35,36]. Through this sort of facilitation, game designers understand the conditions needed for a successful ethical design and allow space for each design to arise out of specific situations. This includes extending the same understanding to users in the context being designed for. For example, a game designer might create a collage of the history of transportation in the city. The player might be asked to do the same. In doing so, not only does the game designer place herself in the global context, but allows for self-reflection and understanding of the differences and commonalities between her values and those of the player.

\section{The Contexts}

In the previous section we discussed the ethical considerations for the design and development of serious games from the perspective of the designer: which questions to ask and what steps to take. We suggested that the design choices that a designer makes during the design process define the serious game. Some of these design choices and ethical dilemmas are similar regardless of the application context. We defined context 
as the environment and circumstances in which the game is designed (context of design) and intended to be used (context of use). However, some particular application contexts present their own ethical discussions, stemming from the nature of that context. From our perspective, the contexts of design and use do not operate in isolation (as Figure 2 shows), rather, they are closely linked to other variables in the ethical ecosystem. In this section, two relationships between contexts and variables are explored. The first is the relationship between context of design and context of use through discussions on education and military games. The second relationship discussed in this section, is the connection between context of use and business ethics in privacy aspects of serious games.

\subsection{Serious Games and Education}

In education, serious games support learning through a variety of methods, such as providing practice-with-feedback scenarios in a motivating environment, integrating learning environments with instruction, adaptation to the learner's individual needs, and integrated assessments. A number of ethical issues in serious games for learning are becoming apparent but are left largely unaddressed.

The goal of learning games is primarily to help a learner achieve a set of learning goals, through interactive experience and real time feedback. Often associated with such educational games is the opportunity to provide individualised or personalised learning, by adapting content to the needs of the learner. The introduction of so-called virtual coaches, with realistic affective behaviour towards the learner, begs the question of whether the goal is becoming to replace traditional teachers with technology. While there is much to say in favor of taking advantage of technology in education, there is an inherent ethical discussion on the appropriate extent of this use.

This closely relates to the variation in educational materials used within a curriculum: how much of a curriculum should be gamified? It is unclear whether game-based learning suits some learners better than others. Thus, as an extension of ethical stewardship in game design for specific courses or learning goals, there is a broader need for stewardship in the design of an educational program as a whole.

Learning with digital tools such as serious games allows the mass collection of data about the learner and the learning process. Such game analytics and learning analytics may be used to monitor progress, provide feedback, and support a learner with personalised feedback. However, the ownership of the collected data is often unclear and outside of the learner's control. Moreover, it can be debated whether personalised learning is always in the best interest of the learner: perhaps a different choice in the automatically selected level of difficulty, learning materials, and exercises may be more effective. There is a possibility that a learner is denied access to educational resources that are made available to other students. 
The goals of a learning game may not always be clear. America's Army is positioned as an entertainment game as well as a training tool; yet if we regard the game from a business ethics perspective, it is not transparent that the intended use of the game is to recruit the best players to work for the U.S. army [37,38]. The average player is likely unaware of that and hence not in a position to consider the implications of this hidden purpose. Laying open agendas of an algorithm and making choices on an algorithm transparent is the goal of an area recently termed IT Algorithm Accountability [39,40].

One of the areas in which serious games for learning are particularly popular is in medical training. Serious games and simulations offer effective environments to practice without real-world consequences, as particularly in this context of use, the cost and impact of a mistake is high. However, the fidelity of the simulation to the real world situation is crucial in providing effective training. If it turns out that using the serious game as a training tool produces unwanted effects, who is responsible for the resulting consequences? For example, a serious game about medical triage procedures is used to train staff in performing the correct steps [41]. If a mistake would be made in actual triage procedures because of a misrepresentation in the serious game, it is unclear where liability falls.

Another area of training in which serious games are being used is in the training of intercultural awareness and communication. For example, in the graphic adventure game It's a Deal!, Spanish men and women in international trade are trained in general awareness of cultural differences, as well as specific rules that apply to British culture [42]. The question is whether the design is accurate: are the scenarios representing the cultures involved correctly, or are there underlying assumptions that may be detrimental in real life situations? Using the Hofstede model of synthetic cultures [43] as the basis for the game play, the game design implicitly assumes that cultures are bounded or binary (e.g. collectivist vs. individualist), whereas the real life situation is more varied and complex. This may cause players to over-generalize based on their training. A similar example can be found in the Adaptive Thinking and Leadership (ATL) training game, a multiplayer simulation game used as part of the training for U.S. Army Special Forces Officers [44]. The accuracy of the scenarios, actions, and feedback within the game have a significant impact on how well the training outcomes transfer to real life situations.

These examples showcase the delicate relationship between context of use and context of design. Designers require an understanding of underlying assumptions that they may have about the context for which they are designing (context of design). Equally critical is reflecting on how these assumptions are transferred to the design of the serious game, and how these then influence the player (context of use). 


\subsection{Serious Games and the Military}

"A military convoy is traveling on a rough desert road in Iraq. Suddenly there is a deafening noise: a Humvee explodes ahead, black smoke rises. Rebels attack the vehicles from all sides - shots are heard, screams fill the night. The smell of burning rubber impregnates the air." [45]

This story illustrates the typical manner in which military serious games present their narratives. In contrast to games for entertainment, these narratives reflect real events. Military games simulate actions which have ramifications in real life; therefore serious games for the military raise ethical issues. This section reviews the ethical issues for digital serious games with military applications. Specifically we present a historical summary of military serious games followed by a look at the application context and the linked cultural aspects; we examine the difference between simulations and games; and we discuss how these computer programs are aligned in the military decision-making process.

In the field of ethics and moral, military serious games reflect multiple variables from our ethical framework (cf. Section 2). In particular we focus on three variables: (a) cultural aspects, (b) business ethics and (c) ethical play. The term military ethics encompasses these variables. Military ethics is typically understood as applied professional ethics and concerns questions regarding the application of force by military armed forces [46].

There is a long connected history between serious games and the military [47], as the first serious games were developed for military training. These games were designed, developed and used by the military, like the U.S. Army, during the Cold War [48]. One of the most famous examples is the arcade game Army Battlezone and its specialized version The Bradley Trainer by Atari in 1980, used by the U.S. Army as a targeting training simulator for a specific tank type. In the game the user views a plane with hostile enemy tanks and mountainous horizon from a first-person perspective. The goal is to target and destroy moving enemy tanks and collect reward points. The game displays the objects with wireframe vector graphics on a black and white screen. The game Battlezone represents one of the early provocations of cultural and moral differences between the military and game developers: several of Atari's employees were opposed to the "game" content and refused to make further games for the military [48]. The original designer of Battlezone, Ed Rotberg, stated: "We didn't want anything to do with the military. I was doing games. I didn't want to train people to kill." [48]. Serious games for the military have since become a huge industry. America's Army, Virtual Battlespace, Steel Beasts or Combat Flight Simulator are recent games with large budgets made for the U.S. military [49].

The narratives and scenarios of games for the military are clear: war or war-like conflicts and their serious ramifications. Hence, those games are not just for entertainment with fictional narratives, but rather have serious application contexts and events. 
Serious games for military train people to ultimately support martial actions. The usage of realistic and violent first-person shooters (FPS) which train people how to kill raises ethical issues. There is empirical data providing evidence that people's personality structures are significantly altered when playing these shooter games [2]. That is, in the short-term, violent games affect aggression by priming aggressive thoughts, and in the long-run the repeated exposure to computer game violence can lead to increases in aggressive affect, which can negatively influence everyday social interactions [2]. Obviously this is of high relevance when such games are directly linked with real war-like application scenarios, like the training of combat situations which include killing actions. Of course, in the military such training is inherently part of the profession itself. However, the ultimate intended outcome of such training is not only for defensive actions but could also lead - directly or indirectly — to offensive attack actions. Again, in the formal military context and with military personnel this is part of the profession. But for a general audience it is questionable whether games which can change the personality structure and could affect aggression are tolerable for society. At this point questions arise whether it is ethical to design and develop games for the military (which are open to the general public) since they can negatively influence multiple people's lives.

An example for a military first-person shooter game is the often-cited recruiting game America's Army, developed and published by the United States Army. It is a subject of ongoing criticism and controversy[37,50]. America's Army is the name of a technology platform used to create free realistic army games permitting (young) Americans to virtually explore typical combat situations with other players in multiplayer scenarios. As a strategic communication device, the game is designed to collect usage data used by the U.S. Army for recruitment purposes. In the same manner, China's People's Liberation Army (PLA) has released the similar first-person shooter game Glorious Mission with the dual purpose of recruiting soldiers and training personnel in combat skills and technological awareness. In some societies such games are generally more or less accepted, whereas in others the topic is highly controversial. In Germany, contentious discussions arose over 3D shooter games which have human-like characters being harmed or killed; the games are often discredited as "Killerspiele" ("killer games") [51]. Of course, there are also military serious games designed for more positive purposes, i.e., which not only concern the application of force but train social skills like inter-cultural communication $[52,53]$ or games for psychiatric rehabilitation targeting post-traumatic stress disorder (PTSD) [45].

In the military, the term simulation is more often used than serious game. The political acceptance level is much higher for serious simulations (even with game-like concepts like narratives etc.) than for computer games which are explicitly declared as such. Typically, no one wants to talk about gaming when it comes to ethically questionable activities. From a technical perspective, there exist distinct differences between 
military computer simulations and entertainment games. In military computer simulations, the focus is not on the explicit display of violence or of harmed bodies, neither the killing action nor the display of use of force from a first person perspective. Military simulations focus primarily on high realism of equipment or processes, and not on the most realistic use of force. Whereas entertainment games have their realism focus on the realistic display of violence (blood detail, weapon details etc.), military simulations focus mostly on correct (realistic) physics or validity of processes. These simulations capture the physics and aerodynamics present in the use of equipment such as weapons, vehicles, tanks or aircraft. Understanding and learning how these work is essential for the training of soldiers to safely operate real, complex equipment.

Often mentioned in discussions of this topic is the analogy to the application of weapons: a gun itself is not dangerous, but the human using it could be. Hence, it is the responsibility of people to make proper use of tools. This is often applied to software as well: the military computer simulation for training itself is at first not directly linked to any killing. But, of course, it could be used to facilitate the process of harming others. For example, in the computer simulation, soldiers could have been trained to steer weaponized military drones, be they manually steered or semi-autonomous $[54,55]$. Those type of weapons enable soldiers to participate in wars and conflicts, but from a very remote location and not in direct, life-threatening contact. It is important that soldiers are also trained to never forget their responsibility for their remotely executed actions. They are always part of the military kill-chain [56]. Localizing a military computer program in the military decision-making process, like the military kill-chain model, is difficult; it depends on the nature of the software, i.e., whether it is an educational serious game or a tactical simulator. An educational serious game (or a computer simulation for just training) could be seen at the very beginning of a military decision process. The educational serious game is right at the front disconnected from any killing action and only used in a formal education context, e.g., training at the military academy. Typically, it is not part of a combat situation, i.e. it is not part of any killing action. In contrast, the link in the kill-chain is much stronger for an operational military tactical simulator which is used right before the soldier continues with the real weaponized drone. In this case, this computer simulator is actually part of the military kill-chain and the ethical issues apply.

The application of military games are multiple and broad, and can include infantry, flight simulators, tanks, submarines, tactics, strategy, trauma management and others $[47,48,49]$. Such applications of military games therefore merit an exploration of the ethical issues of their use. As long as there are conflicts, the military and its industrial partners will continue to produce military serious games for recruitment, training, and education. Those games will always be subject to the aforementioned ethical issues. As researchers and designers of serious games it is important to critically question their proper and ethical use since serious games for military use appear to be permanently embedded in society, for now. 


\subsection{Serious Games and Data Privacy}

With the number of users and strategies for sharing and storing data about them increasing, data privacy becomes an important issue in serious games design. However, there is no common definition of the term data privacy. In general, it refers to the efforts to control any access of user-related data by any third party. Video games can be considered rich origins of data: they are interactive software systems by design. Games receive input data via various channels such as interactive interfaces and controllers, which potentially can be collected and stored digitally by the game software. The following example demonstrates how much data is produced by games. Eggert et. al. [57] exploit replay files of a real time strategy game to classify player types, using behavioral lowlevel data during game play. Even if replay files may not be stored by default, it demonstrates the ability of the game software to log each small game interaction, which is sufficient to reproduce a complex game play. Another kind of data available for use are player stats - data that defines the current status of the player in the game. The massively multiplayer online game (MMOG) EVE Online [57] serves as an example. EVE Online contains a comprehensive data model - a great amount of game related (player status) data has to be managed. For example in the market a player has submitted offers and bids. Player skills have to be developed in order to gain certificates, therefore optimized schedules of (time-based) skill training have to be developed. There is even a data interface, which has led to the emergence of a number of third party tools with the purpose of user-friendly administration of game-related data [58]. Thus, video games collect, aggregate and store large amounts of player data.

Whenever data is collected and stored, there is the potential for misuse. In general, data protection laws have been enacted in order to protect data privacy. However, those laws do not provide regulations specific to video games. In the context of video games, misuse could be defined as using game data for other purposes than entertainment by this game. Serious games are by definition videogames with further purposes besides pure entertainment, thus such a definition seems to be insufficient in the context of serious games. Those further purposes might require collection and processing of even more user-related data. For example, in adaptive learning games, the current user status has to be stored. In the learning game Doctor's Cure [59], students assume the role of a reporter investigating the details of a moral dilemma narrative hint by hint. Collected data from measuring learning progress is provided to the teacher through the Teacher Dashboard, and the teacher takes on the role of editor in communicating with the students. So, in order to support the goals of the game, there is an inherent requirement to generate and store additional data, i.e., the information needed in the Dashboard and the communication log between student and teacher.

For a serious game this definition has to be extended: data should be used only for this game's purposes - including and besides entertainment. However, the definition continues to seem incomplete, as the following examples should illustrate. Fliplife [60] 
is a social network game ( $\mathrm{SNG}$ ), used also as a storytelling platform for companies, making it a so-called advergames, a kind of a serious game. In Fliplife aspects of daily life are simulated: work, education and spare-time. The player chooses a career she wants to pursue and works on it in projects, which are timer-based activities, together with other players, the co-workers. Careers are provided by companies in order to implement a narrative corporate presentation in the game. Furthermore, a German trust has been said to consider the game as as a source for potential employees [61]: based on game-collectible data, a list of candidates for job interviews can be generated. From a methodological point of view such an attempt to identify candidates for a job can be considered as completely feasible [62]. From an ethical point of view, there remain at least doubts: players are not explicitly aware of the collection of data which does not support game mechanics, but enables third parties to trace their personality traits like reliability, engagement or leadership qualities of players. Using the proposed ethical ecosystem, this kind of data exploitation falls under the category of Business Ethics and can be a part of a business model for the game supplier. Observing players in this way appears unproductive if they are aware of this surveillance and act accordingly. Consequently, this collection and use of data should not be revealed to players. Then it becomes hidden surveillance, which is ethically problematic. Another example in this context of the ethical ecosystem in the category Business Ethics is the smartphonebased augmented reality game INGRESS [63]. This game requires gamers to walk around in the neighbourhood and visit locations with virtual portals in order to interact with those portals virtually. During the game play, a great deal of data could be collected, which would be useful in other contexts [64]. So for example, preferred routes and activity times could be tracked. Much of that data is already available at mobile providers, but now it would be exploitable for the game provider at a much more detailed level. Among the positive imaginable results of player's actions are pictures with attached position data, which could augment online map services and WLAN identifiers. These artifacts may be highly useful for tracking services. Actually, such a use is denied by the game developer [65], although it is not explicitly excluded by the Terms of Service and Privacy Rules [66]. Therefore, there is a high likelihood that a sudden change of data usage would go unnoticed by users.

Although the importance of data privacy is valued differently across countries and cultures, the disclosures of Edward Snowden [67] have raised it to wide public attention. The increasing use of online games and mobile apps further establishes the relevance of data privacy in the context of games. A recent German study about data privacy in online games "found several breaches of data protection rules. Most of the privacy policy statements did not meet the required strict German standard of comprehension and completeness" [68]. Further, the study claims data privacy is handled heterogeneously, mostly depending on the country where the servers are based. On the other hand, the results indicate that "many users are interested in data protection and privacy issues." 
As there are no widely accepted standards, and as it seems that such a standard will not be established in the near future, serious game developers must proactively address data privacy responsibly, i.e., carefully taking ethical considerations into account. As already indicated, in the context of serious games, data privacy is a matter of Business Ethics based on the above defined variables of the ethical ecosystem. As serious games are intended to pursue further purposes than pure entertainment, the needs for these purposes guide the development and application of those games. At the same time, these purposes limit the degrees of freedom regarding design and implementation of a serious game. "Enjoyment" is the goal which must be achieved by pure entertainment games; for serious games, entertainment and enjoyment are employed, but not for the primary purpose of the games. Therefore, within the categories of our proposed Ethical Ecosystem (see Figure 2), Business Ethics might be the dominant variable, as the examples of Fliplife and INGRESS illustrate. There might be an inherent drive to the exploitation of generated data, which has to be reviewed in its ethical dimensions carefully and leads to data privacy being an ethical matter. However, the three central elements of the Ethical Ecosystem can be used to categorise the concerns of data privacy. Mainly, The Serious Game provides a frame for adherence to ethical implementation of data privacy concerns. Furthermore, The Designer is in charge of negotiating a reasonable balance of data privacy requirements and serious games goals, and The Player should be made aware of the privacy policies implemented in the game

Responsible data privacy at least comprises implementing national data protection laws of those countries where the game is rolled out, and revealing clearly and precisely the actual procedures of collection, storage and use of data in a meaningful data policy. The user should be informed about the current ways to utilize the game's data. Even if the issue of data privacy is raised by quite a few games (e.g., Data Dealer [69] or Privacy Pirates [70]) there is no commonly accepted guideline. This missing regulation may endanger the acceptance of (serious) online games in the long term: if players hesitate to play a game due to privacy concerns, this would be an unnecessary obstacle of the game developers' own doing. All the more, game developers are required to adhere to a clear and responsible data privacy guideline.

\section{Conclusions and Outlook}

The purpose of this chapter is to highlight the importance of moral intelligence in game development with a special focus on serious games. To support moral intelligence in game development, we proposed an Ecosystem for Designing Games Ethically (EDGE). The ethical ecosystem integrates the work of previous scholars such as Zagal and Flanagan into one system that includes the designer and the contexts of use as necessary to ethical reflection. We defined these contexts of design and use. The context of design is one in which the designer makes the design choices that define the game and the context of use is one within which the player interacts with the game. Context 
considerations for specific design contexts were reviewed including contexts of educational games, and games with military game play. Data privacy considerations were also included.

In addition to EDGE, we provided a process for serious game designers to implement moral intelligence in design which we termed, Ethical Stewardship (ES). A primary aim of ES is to strengthen relationships between designers, players and stakeholders throughout the entire process of moral competence in designers. Ethical stewardship does not negate the business of digital games-- the need for institutions, or game studios to be profitable, nor the practices required to expand serious games to new markets. Ethical stewardship also does not blindly affirm that every ES guideline must be met for moral intelligence. We acknowledge that constraints exist in the development process and that it is not always possible to include all components of ES. Rather, we offer ES as a way to embrace ethical ethnorelativism and moral design in serious games. In practice, ethical stewardship can be addressed through education and training. The myriad of university curricula addressing the design and development of serious game bear the responsibility to actively address ethical design considerations in their projects.

In closing, we wish to contribute some remarks on the importance of moral intelligence in digital game design. As socially constructed artifacts, digital games influence social, psychological, political and economic contexts $[8,71]$. Several design perspectives assume that embracing ethical competence is vital for good design [32,35]. Some argue that game designers, as creators of art, media and cultural artifacts, have a moral and social obligation to understand the impact their design choices have on societythat there are consequences to what is designed and therefore consumed by others $[19,40,72,73,74]$. Others argue that self-reflection in design is essential for personal and professional growth. Deardorff [23,24] and others [75] claim that embracing moral intelligence is an important part of human development and of "good" citizenship. Alternatively, it could be said that neglecting "ethics" contributes to the lack of moral intelligence in game creation, and that in itself undermines the success of serious design efforts and contributes to questionable design decisions such as those in H2ST [32].

Instead, this chapter's authors assert that ethical stewardship ought to be embarked upon because richness of artifact creation - the creation of new forms of serious games - lies in the constraints an ethical stance affords. Ethical stewardship is not simply a methodology for moral competence as good action or self-growth. Nor a method to pursue what others identify as good design. Rather, ethical stewardship makes a commitment to reflecting upon moral intelligence as critical to creativity and innovative action. Greater moral competence in design ultimately results in a more integrated player experience for its respective market, improved game content quality and stronger partnerships with stakeholders. At a larger scale, ethical considerations need to become standard practice in game design and development frameworks and a topic of discussion in the respective academic journals and conferences, as well as in the field of practice. 


\section{$6 \quad$ Reflective Questions}

- What challenges present themselves to the designer who wishes to include an ethical perspective?

- What ethical considerations would be most difficult to integrate and why?

- In what manner can the suggested tools be integrated in your design process to develop alternative perspectives and critical reflection?

- Should all games require an ethical certification or course? Which usages of playerrelated data can be considered as ethically appropriate?

- What impact has a clear data privacy term on the usage of a serious game in comparison to the absence of such terms?

- What recent developments in technology require new approaches in data privacy (e.g., health data [63], [64], location based data, and route tracking)?

- How to systematically assess the influence of serious games designed for specific contentious purposes (e.g. military)? How to define an ethical assessment framework?

\section{$7 \quad$ Further Reading}

To develop a deeper understanding of the perspectives mentioned in this chapter, the following list is suggested for further reading:

Values in game design: Flanagan, M., \& Nissenbaum, H. (2014). Values at play in digital games. Boston, MA: MIT Press.

Information ethics: Floridi, L. (Ed.). (2010). The Cambridge handbook of information and computer ethics. Boston, MA: Cambridge University Press.

Digital game ethics and models: Schrier, K. (Ed.). (2010). Designing games for ethics: Models, techniques and frameworks. Hershey, PA: IGI Global.

Player ethics: Sicart, M. (2011). The ethics of computer games. Boston, MA: MIT Press.

Digital game ethics: Zagal, J. P. (2011). The videogame ethics reader. San Diego, CA: Cognella Academic Publishing.

\section{Acknowledgement}

In addition to Prof. Katharina-Anna Zweig (zweig@cs.uni-kl.de), our chapter editor, we would like to thank Dr. Cheri Gurse, founder of Scholar Builder, for her time and dedication in editing this chapter. Dr. Gurse works as a professional editor in the Social Sciences and can be reached at cgurse@fielding.edu 


\section{Bibliography}

1. M. Sicart, "The banality of simulated evil: designing ethical gameplay," Ethics Inf. Technol., vol. 11, no. 3, pp. 191-202, 2009.

2. C. A. Anderson and K. E. Dill, "Video games and aggressive thoughts, feelings, and behaviour in the laboratory and in life.," J. Pers. Soc. Psychol., vol. 78, no. 4, p. 772, 2000.

3. J. L. Sherry, K. Lucas, B. S. Greenberg, and K. Lachlan, "Video game uses and gratifications as predictors of use and game preference.," Play. video games Motiv. Responses Consequences, pp. 213-224, 2006.

4. J. A. Vessey and J. E. Lee, "Violent video games affecting our children.," Pediatr. Nurs., vol. 26, no. 6, pp. 607-609, 2000.

5. J. P. Zagal, "Ethically Notable Videogames: Moral Dilemmas and Gameplay," in Proceedings of DiGRA 2009: Breaking new ground: Innovation in games, play and practice and theory, 2009, p. 9.

6. T. W. Bynum, "Computer ethics: Basic concepts and historical overview," in The Stanford Encyclopedia of Philosophy, 2001.

7. T. W. Bynum, "Flourishing ethics," Ethics Inf. Technol., vol. 8, no. 4, pp. 157-173, 2006.

8. M. Flanagan, H. Nissenbaum, D. C. Howe, and H. Nissenbaum, Embodying values in technology: Theory and practice. Cambridge University Press, 2008.

9. A. Shaw, "What is video game culture? Cultural studies and game studies.," Games Cult., vol. 5, no. 4, pp. 403-424, 2010.

10. M. Flanagan and H. Nissenbaum, Values at Play in Digital Games. MIT Press, 2014.

11. A. Sandovar and D. L. V. Street, "Cultural Narratives in Game Design," no. Fdg, pp. 4-6, 2015.

12. J. R. Whitson, "Game Design by Numbers: Instrumental Play and the Quantitative Shift in the Digital Game Industry," Carleton University, Ottawa, 2012.

13. C. O'Donnell, Developer's Dilemma: The secret world of videogame creators. Cambridge, MA: MIT Press, 2014.

14. B. Friedman, P. Kahn, and A. Borning, "Value sensitive design: Theory and methods," 2002.

15. G. Cockton, "Designing worth - connecting preferred means to desired ends.," Interact. $A C M$, vol. July/Augus, pp. 54-57, 2008.

16. M. Flanagan, D. C. Howe, and H. Nissenbaum, "Values at Play: Design Tradeoffs in Socially-Oriented," Chi 2005, pp. 751-760, 2005.

17. N. Manders-Huits and M. Zimmer, "Values and pragmatic action: The challenges of introducing ethical intelligence in technical design communities," Int. Rev. Inf. Ethics, vol. 10, pp. 37-44, 2009.

18. C. Ess, Digital media ethics. Cambridge, UK: Polity Press, 2009.

19. H. Nissenbaum, "Values in the design of computer systems," Comput. Soc., vol. 28, no. 1, pp. 38-39, 1998.

20. M. Battiste, "Indigenous knowledge: Foundations for first nations," 2005.

21. R. Rath, "The Historical Case for Playable Women in Assasin's Creed: Unity," The Escapist, 2014.

22. S. Leboeuf, "Editorial: Omitting Women From Games Because 'It's Too Hard' is Unacceptable," The Escapist, 2014.

23. D. K. Deardorff, “Assessing intercultural competence," New Dir. Institutional Res., vol. 149, pp. 65-79, 2011.

24. D. K. Deardorff, "Identification and assessment of intercultural competence as a student outcome of internationalization,” J. Stud. Int. Educ., vol. 10, no. 3, pp. 241-266, 2006. 
25. C. Taylor and S. Dempsey, "Ethics Quest: Harnessing the Power of an RPG to Help Interdisciplinary Teams," in Presented at the Games for Change Conference, 2016.

26. B. Hannington, "Methods in the making: A perspective on the state of human research in design," Des. Issues, vol. 19, no. 4, pp. 9-18, 2003.

27. M. Kamppuri, M. Tedre, and M. Tukiainen, "Towards the sixth level in interface design: Understanding culture," in Proceedings of the CHI-SA, 2006, pp. 1-6.

28. C. Lévi-Strauss, The savage mind. Chicago: University of Chicago Press, 1967.

29. S. B. Merriam and Y. S. Kim, "Non-western perspectives on learning and knowing," New Dir. adult Contin. Educ., vol. 119, pp. 71-81, 2008.

30. A. O. Asojo, "A model for integrating culture-based issues in creative thinking and problem solving in design studios.," J. Inter. Des., vol. 27, no. 2, pp. 46-58, 2001.

31. B. Grant, "Cultural invisibility: The African American experiences in architectural education," in Voices in tribal education: cultural politics and pedagogy, T. A. Dutton, Ed. New York: Bergin \& Garvey, 1991, pp. 149-164.

32. K. Edwards, "Culturalization: The Geopolitical and Cultural Dimension of Game Content," Trans, vol. 15, pp. 19-28, 2011.

33. A. Acharya and B. Buzan, Eds., Non-Western international relations theory: perspectives on and beyond Asia. Routledge, 2009.

34. J. Czarnota, "Designing player-studio interactions for co-creation: Reviewing academic theory with industrial practice.," in Multi.Player 2 Conference Abstract, 2014.

35. H. Winschiers-Theophilus, "The Art of Cross-Cultural Design for Usability," in Universal Access in HCI, Part 1, C. Stephanidis, Ed. LNCS 5614, 2009, pp. 665-671.

36. J. Lee and S. Sayed, "Culturally sensitive design," in Design connections: Knowledge, value and involvement through design, Y. Levanto, P. Sivenius, and S. Vihma, Eds. 2008, pp. 54 63.

37. N. Turse, “Zap, Zap, You're Dead...," TomDispatch.com, 2003. [Online]. Available: http://www.tomdispatch.com/post/1012/.

38. C. Morris, "Your Tax Dollars at Play," CNN Money, 2002.

39. N. Diakopoulos, "Algorithmic Accountability - Journalistic Investigation of Computational Power Structures," Digit. Journal., vol. 3, 2015.

40. N. Diakopoulos, "Accountability in Algorithmic Decision Making," Commun. ACM, vol. 59, pp. 56-62, 2016.

41. E. D. van der Spek, Experiments in serious game design: a cognitive approach, vol. 201136. 2011.

42. V. Guillén-Nieto and M. Aleson-Carbonell, "Serious Games and Learning Effectiveness: The Case of It's a Deal!," Comput. Educ., vol. 58, no. 1, pp. 435-448, 2012.

43. G. J. Hofstede, P. B. Pedersen, and G. Hofstede, Exploring Culture: Exercises, Stories and Synthetic Cultures. Yarmouth, ME: Intercultural Press, 2002.

44. E. M. Raybourn, E. Deagle, K. Mendini, and J. Heneghan, "Adaptive Thinking \& Leadership Simulation Game Training for Special Forces Officers," in Interservice/Industry Training, Simulation and Education Conference (I/ITSEC)., 2005.

45. A. Rizzo, "Bravemind: Virtual Reality Exposure Therapy," University of Southern California Institute for Creatrive Technologies, 2005.

46. M. L. Cook and H. Syse, "What Should We Mean by 'Military Ethics'?," J. Mil. Ethics, vol. 9, no. 2, pp. 119-122, 2010.

47. M. Prensky, "True Believers: Digital Game-Based Learning in The Military," Digit. GameBased Learn., pp. 1-18, 2001. 
48. S. Kent, The Ultimate History of Video Games: From Pong to Pokemon and Beyond... The Story Behind the Craze that Touched our Lives and Changed the World. Three Rivers Press, 2010.

49. P. A. Roman and D. Brown, "Games - Just How Serious Are THey," in The Intersevice/Industry Training, Simulation \& Education Conference (I/ITSEC), 2008.

50. M. Schulzke, "Rethinking Military Gaming: America's Army and its Critics," Games Cult., vol. 8, no. 2, pp. 59-76, 2013.

51. P. Nauroth, J. Bender, T. Rothmund, and M. Gollwitzer, "Die 'Killerspiele'-Diskussion: Wie Die Forschung Zur Wirkung Gewalthaltiger Bildschirmspiele in Der Öffentlichkeit Wahrgenommen Wird.," in Neue Medien Und Deren Schatten. Mediennutzung, Medienwirkung Und Medienkompetenz., T. Porsch and S. Pieschl, Eds. Göttingen: Hogrefe, 2014, pp. 81-100.

52. Buch, Tascha, and S. Egenfleldt-Nielsen, "The Learning Effects of Global Conflicts: Palestine,"in Medi@, Terra Conference, 2006.

53. W. L. Johnson, N. Wang, and S. Wu, "Experience with Serious Games for Learning Foreign Languages and Cultures," in Proceedings of the SimTeT Conference, 2007.

54. P. Lin, G. Bekey, and K. Abney, "Autonomous Military Robotics: Risk, Ethics, and Design," 2008.

55. J. Borenstein, "The ethics of autonomous military robots," Stud. Ethics. Law. Technol., vol. 2, no. $1,2008$.

56. R. Azuma, M. Daily, and C. Furmanski, "A Review of Time Critical Decision Making Models and Human Cognitive Processes," in IEEE Aerospace Conference, 2006.

57. C. Eggert, M. Herrlich, J. Smeddinck, and R. Malaka, "Classification of Player Roles in the Team-Based Multi-player Game Dota 2," Entertain. Comput. ICEC, pp. 112-125, 2015.

58. CCP, "3rd Party Tools," EVElopedia, 2015. [Online]. Available: https://wiki.eveonline.com/en/wiki/3rd party tools.

59. A. Arici and S. Barab, "Transformational Play; Using 3D Game-Based Narratives to Immerse Students in Literacy Learning," in European Conference on Games Based Learning, 2013, p. 35.

60. "Fliplife." 2012. [Online, (Game discontinued 30. Sept. 2014)]. Available: http://fliplife.com/..

61. M. Meyer, "Per Spiel zum Traumjob?," FORUM - Das Wochenmagazin, 2011. [Online]. Available: https://web.archive.org/web/20111130094810/http://www.magazinforum.de/per-spiel-zum-traumjob.

62. H. Söbke, C. Hadlich, N. Müller, T. Hesse, C. Henning, S. Schneider, and O. Kornadt, "Social game fliplife: digging for talent - an analysis," in Proceedings of the 6th European Conference on Games Based Learning, 2012, pp. 487-494.

63. Niantic Labs, "Ingress.” Google, 2013. [Online]. Available: http://www.ingress.com/.

64. S. Lobo, "S.P.O.N. - Die Mensch-Maschine: Google macht die Welt zum Spielfeld," Spiegel Online, 2013. [Online]. Available: http://www.spiegel.de/netzwelt/web/google-ingress-dieganze-welt-als-spiel-a-902267.html.

65. J.-K. Janssen, “Ingress-Erfinder: 'Visionär zu sein ist uns wichtiger als Datensammeln'" c't Mag. für Comput., no. 15, p. 41, 2014.

66. "Ingress Terms of Service," Niantic, Inc., 2015. [Online]. Available: https://www.ingress.com/terms.

67. G. Greenwald, "No Place to Hide: Edward Snowden, the NSA, and the U.S. Surveillance State," Picador. 2015.

68. "Unabhängiges Landeszentrum für Datenschutz Schleswig-Holstein (ULD)," 2010. [Online]. Available: https://www.datenschutzzentrum.de/projekte/dos/results/. 
69. Cracked Labs, "Data Dealer. Legal? Illegal? Whatever." 2013. [Online]. Available: http://datadealer.com/.

70. "Privacy Pirates: An Interactive Unit on Online Privacy." MediaSmarts, 2015. [Online]. Available: http://mediasmarts.ca/sites/mediasmarts/files/games/privacy_pirates/flash/PrivacyPirates_English/main.html.

71. A. Kerr, "The culture of gamework," in Managing Media Work, M. Deuze, Ed. London: Sage, 2011.

72. M. Akrich, Beyond social construction of technology: the shaping of people and things in the innovation process. Campus/Westview, 1992.

73. M. Flanagan, J. Belman, H. Nissenbaum, and J. Diamond, "A method for discovering values in digital games," in Paper presented at Situated PLay DiGRA '07, Tokyo, Japan, 2007.

74. J. Van den Hoven, Information technology and moral philosophy. 2008.

75. B. H. Spitzberg and G. Changnon, "Conceptualizing intercultural competence," in The Sage Handbook of Intercultural Competence, Thousand Oaks, CA: Sage, 2009, pp. 2-52. 